\title{
Association of Automatically Quantified Total Blood Volume after Aneurysmal Subarachnoid Hemorrhage with Delayed Cerebral Ischemia
}

\author{
(DI.A. Zijlstra, (D) C.S. Gathier, (D)A.M. Boers, (D) H.A. Marquering, (D)A.J. Slooter, (DB.K. Velthuis, (D) B.A. Coert, (D)D. Verbaan, \\ (1D) van den Berg, (D) G.J. Rinkel, and (D) C.B. Majoie
}

\begin{abstract}
BACKGROUND AND PURPOSE: The total amount of extravasated blood after aneurysmal subarachnoid hemorrhage, assessed with semiquantitative methods such as the modified Fisher and Hijdra scales, is known to be a predictor of delayed cerebral ischemia. However, prediction rates of delayed cerebral ischemia are moderate, which may be caused by the rough and observer-dependent blood volume estimation used in the prediction models. We therefore assessed the association between automatically quantified total blood volume on NCCT and delayed cerebral ischemia.
\end{abstract}

MATERIALS AND METHODS: We retrospectively studied clinical and radiologic data of consecutive patients with aneurysmal SAH admitted to 2 academic hospitals between January 2009 and December 2011. Adjusted ORs with associated 95\% confidence intervals were calculated for the association between automatically quantified total blood volume on NCCT and delayed cerebral ischemia (clinical, radiologic, and both). The calculations were also performed for the presence of an intraparenchymal hematoma and/or an intraventricular hematoma and clinical delayed cerebral ischemia.

RESULTS: We included 333 patients. The adjusted OR of total blood volume for delayed cerebral ischemia (clinical, radiologic, and both) was 1.02 (95\% Cl, 1.01-1.03) per milliliter of blood. The adjusted OR for the presence of an intraparenchymal hematoma for clinical delayed cerebral ischemia was $0.47(95 \% \mathrm{Cl}, 0.24-0.95)$ and of the presence of an intraventricular hematoma, $2.66(95 \% \mathrm{Cl}, 1.37-5.17)$.

CONCLUSIONS: A higher total blood volume measured with our automated quantification method is significantly associated with delayed cerebral ischemia. The results of this study encourage the use of rater-independent quantification methods in future multicenter studies on delayed cerebral ischemia prevention and prediction.

ABBREVIATIONS: $a \mathrm{OR}=$ adjusted odds ratio; $\mathrm{aSAH}=$ aneurysmal subarachnoid hemorrhage; $\mathrm{DCl}$ = delayed cerebral ischemia; $\mathrm{IPH}=$ intraparenchymal hematoma; IVH = intraventricular hematoma; TBV = total blood volume; WFNS scale = World Federation of Neurosurgical Societies scale

D elayed cerebral ischemia (DCI) occurs in $20 \%-30 \%$ of patients with aneurysmal subarachnoid hemorrhage (aSAH) and is associated with poor outcome. ${ }^{1-3}$ Patients who develop DCI need costly intensive care. The cause of DCI is multifactorial,

Received October 27, 2015; accepted after revision February 7, 2016.

From the Departments of Radiology (I.A.Z, A.M.B., H.A.M, R.v.d.B., C.B.M.), Neurosurgery (B.A.C., D.V.), and Biomedical Engineering and Physics (A.M.B., H.A.M.), Academic Medical Center, Amsterdam, the Netherlands; Departments of Radiology (B.-K.V.), Intensive Care (A.J.S.), and Neurology (C.S.G., G.J.R.), University Medical Center Utrecht, Utrecht, the Netherlands; and Department of Robotics and Mechatronics (A.M.B.), University of Twente, Enschede, the Netherlands.

H.A.M. received a research grant from Fonds NutsOhra (1403-023) to study the prognostic value of quantified blood in patients with aneurysmal subarachnoid hemorrhage.

Paper previously presented at: Annual Meeting of the European Congress of Radiology, March 2-6, 2016; Vienna, Austria.

Please address correspondence to IJsbrand A. Zijlstra, MD, Department of Radiology, Academic Medical Center, Meibergdreef 9, 1105 AZ, Amsterdam, the Netherlands; e-mail ij.a.zijlstra@amc.uva.nl

http://dx.doi.org/10.3174/ajnr.A4771 including larger and smaller vessel vasospasm, cortical spreading ischemia, microvascular dysfunction, and thrombosis. ${ }^{3}$ Bloodbreakdown products in the subarachnoid and CSF spaces may cause vasospasm. ${ }^{2}$ Several studies have reported the positive relationship between the total amount of extravasated blood after $\mathrm{SAH}$ and the development of vasospasm (at that time considered the main cause of DCI) by using CT grading scales such as the modified/revisited Fisher grading scale and the Hijdra scale. ${ }^{4-7}$ These grading scales only provide a rough estimation of the aneurysmal total blood volume (TBV) and are observer-dependent, factors that may add to the moderate prediction rates of DCI. ${ }^{8}$ More reliable quantification of TBV might result in better prediction of DCI, which can help clinicians more accurately identify patients at risk and more effectively use scarce resources. ${ }^{9}$ To assess the association of TBV with DCI, a reliable and valid method for measuring subarachnoid blood volume is needed, with correction for possible confounding influences. We recently validated a fully automatic method for TBV quantification on NCCT. 
This method is based on a relative density increase of blood after aSAH in relation to different brain structures. ${ }^{10}$ In the current study, we aimed to assess the association of automatically quantified TBV with DCI.

\section{MATERIALS AND METHODS \\ Patient Population}

We included consecutive patients with aSAH who were admitted between January 2009 and December 2011 to 2 large university hospitals (Academic Medical Center Amsterdam and University Medical Center Utrecht) in the Netherlands.

"Aneurysmal SAH" was defined as an aneurysmal bleeding pattern with an associated aneurysm. Patients without aneurysms proved on CTA/MRA/DSA were excluded. We further excluded patients with a baseline CT obtained $>24$ hours after ictus because of the risk of blood clearance and patients in whom the CT scans could not be used for the automatic quantification method because of movement artifacts or metal artifacts caused by previous treatment. Patients with technically inadequate scans (scans in 2 parts or incomplete scans) were also excluded. Patients with an external ventricular drain on the first CT were excluded because of artifacts and possible blood clearance. TBV was defined as the sum of subarachnoid (cisternal and sulcal), intraparenchymal, intraventricular, and subdural blood.

\section{Clinical and Imaging Data Collection}

All baseline characteristics of the included patients were collected through retrospective review of the clinical charts by a single observer (C.S.G.). These included age, medical history of hypertension, date of the aSAH ictus, hospital admission date, clinical condition on admission according to the World Federation of Neurological Societies scale (WFNS), ${ }^{11}$ NCCT date, the occurrence of rebleeding (clinical, not CT-confirmed, or radiologic), aneurysm location (anterior or posterior circulation), aneurysm treatment (coiling, clipping, or no aneurysm treatment), and date of death. The WFNS score on admission was dichotomized into favorable (1-3) and unfavorable (4-5). This observer also assessed the presence of clinical DCI, which was defined as clinical deterioration that could not be explained by any cause other than DCI, and radiologic DCI, which was defined as the presence of cerebral infarction on CT or MR images within 6 weeks after SAH or on the latest CT scan or MR image obtained before death, which could not be attributed to other causes such as surgical clipping or endovascular treatment, according to previously published criteria. ${ }^{12}$ The patients were followed as long as they were hospitalized.

For the volume analysis, generally the first CT scan (all 5-mm sections) after the aSAH ictus was used. Only in case of a rebleeding within 24 hours and before treatment was the CT scan after rebleeding used because of the larger blood volume. All CT scans were anonymized before assessment (H.A.M. and I.A.Z.) and were thereafter assessed for the appropriateness for automated quantification (I.A.Z.). The TBV (in milliliters) was automatically quantified (Fig 1), and the quality of the automatic segmentation was evaluated by using ITK-SNAP, Version 2.4.0 (www.itksnap. org) (I.A.Z.). ${ }^{13}$

All CT scans were inspected for the presence of intraparenchy- mal hematoma (IPH) and/or intraventricular hematoma (IVH) (I.A.Z.). The presence or absence of IPH and IVH was scored dichotomously because it was not possible to measure blood volume in separate locations with our automated quantification method. The differentiation between a blood clot in the Sylvian fissure and an IPH was made on the initial CTA (I.A.Z.). ${ }^{14}$ All estimates of blood volume and blood location were performed blinded to the presence or absence of DCI.

\section{Statistical Analysis}

Descriptive Statistics. Dichotomous variables were presented as percentages. Continuous variables were tested with the ShapiroWilk test for normal distribution ( $W>0.9$ is considered a normally distributed variable). Normally distributed variables were expressed as means with SDs, and not normally distributed variables were expressed as medians with interquartile ranges $(25 \%-$ $75 \%)$. Normally distributed variables were tested with the Student $t$ test, and not normally distributed variables were tested with the Mann-Whitney $U$ test. Categoric variables were tested by using the Fisher exact test.

Modeling. Logistic regression analysis was used to calculate odds ratios with associated 95\% confidence intervals. Bivariable analyses were performed with previously chosen covariables known to be associated with DCI on the basis of the literature to identify important confounders (defined as variables that changed the crude OR from the univariable analysis by $>10 \%) .{ }^{15}$ In the multivariable analysis, confounders were added to the univariable model to calculate adjusted odds ratios (aORs) with associated 95\% CIs.

As our primary analysis, we assessed the association between TBV and clinical DCI (with or without radiologic DCI), radiologic DCI (with or without clinical DCI), and clinical and radiologic DCI (patients with both clinical and radiologic DCI) combined. Evaluated confounders were age, sex, neurologic status on admission (dichotomized WFNS grade), treatment of the aneurysm (clipping/coiling/no treatment), rebleeding, hypertension, $\mathrm{IPH}$, and IVH.

As a secondary analysis, we assessed the association between blood location (IPH and IVH) and clinical DCI (with or without radiologic DCI). We used clinical DCI as the only outcome variable because we found similar aORs for all 3 outcome variables (clinical DCI, radiologic DCI, and both) in the primary analysis. Evaluated confounders were age, sex, neurologic status on admission (dichotomized WFNS grade), treatment of the aneurysm (clipping/coiling/no treatment), rebleeding, hypertension, blood volume, IPH (in the model with IVH as the central determinant), and IVH (in the model with IPH as the central determinant).

Because patients who die within 3 days after the aSAH ictus have a much lower risk of developing DCI, we performed sensitivity analyses in the subset of patients who survived $>3$ days after aSAH.

\section{RESULTS}

\section{Patient Characteristics}

We initially evaluated 458 potentially eligible patients with aSAH. Of these, 333 patients were included in the analyses (Fig 2). The 

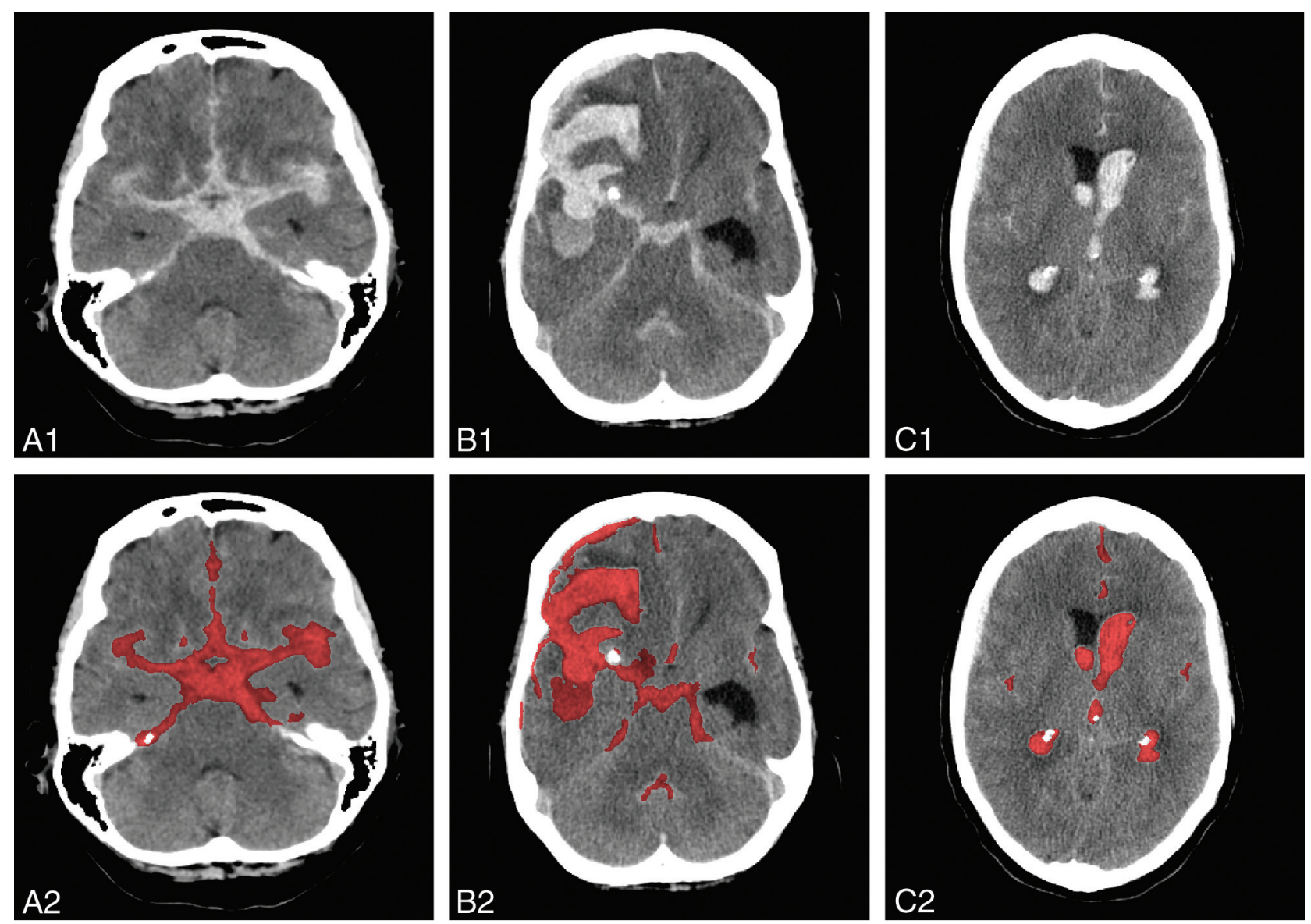

FIG 1. Examples of SAH bleeding patterns on CT (upper), with corresponding segmentations in red as provided by the automatic quantification method (lower). ${ }^{10} \mathrm{~A}$, SAH with blood in both Sylvian fissures. B, SAH with the presence of IPH. C, SAH with the presence of IVH.

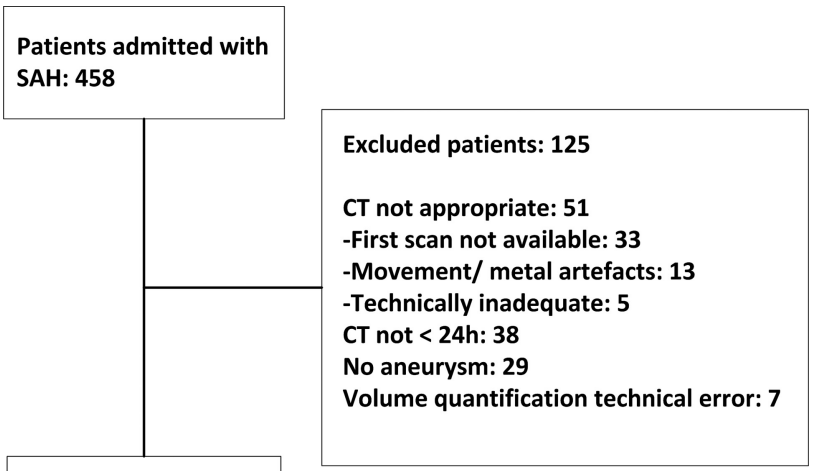

Patients included for

analyses: $\mathbf{3 3 3}$

FIG 2. Flow chart of patient inclusion.

mean TBV was $46.1 \pm 29.4 \mathrm{~mL}$. Characteristics of the included patients are shown in Table 1.

Sixty-eight (20\%) patients had clinical and/or radiologic DCI, 62 (19\%) had clinical DCI (with or without radiologic DCI), 40 (12\%) had radiologic DCI (with or without clinical DCI), and 34 (10\%) had both clinical and radiologic DCI. Twenty-eight (8\%) patients had only clinical DCI, and $6(2 \%)$ had only radiologic DCI (Table 2). One (2.1\%) patient developed clinical signs of DCI 2 days after the aSAH ictus. There were 102 patients (31\%) with IPH, 203 patients $(61 \%)$ with IVH, and 63 patients (19\%) with
IPH and IVH combined. Forty-seven (14\%) patients died within 3 days of the aSAH ictus. The mean TBV in these patients was $63.0 \pm 34.1 \mathrm{~mL}$.

\section{Association between Blood Volume and Blood Location and DCI in the Total Group}

The aOR (95\% CI) of TBV and DCI (clinical, radiologic, both) was 1.02 (1.01-1.03) per milliliter of blood (Table 3$)$. The aOR (95\% CI) of IPH and clinical DCI was $0.47(0.24-0.95)$ and of IVH and clinical DCI, 2.66 (1.37-5.17).

\section{Sensitivity Analysis}

In the 286 patients included in the sensitivity analyses, the mean TBV was $43.3 \pm 27.6 \mathrm{~mL}$. In the subgroup of patients without clinical DCI, the mean TBV was $40.8 \pm 27.2 \mathrm{~mL}$, and in the subgroup of patients with clinical DCI, $52.3 \pm 27.5 \mathrm{~mL}(P=.004)$. IPH occurred in 85 (30\%) patients; IVH, in $172(60 \%)$ patients. The association results in the sensitivity analysis (Table 4) are similar to the results in the total group (Table 3).

\section{DISCUSSION}

In this study, a higher TBV, quantified with a fully automated method, was significantly associated with the development of DCI. The presence of an intraventricular hematoma was also positively associated with the development of DCI, whereas the pres- 


\begin{tabular}{|c|c|c|c|c|}
\hline & $\begin{array}{c}\text { Total Patient } \\
\text { Group (\%) }\end{array}$ & $\begin{array}{l}\text { Patients with } \\
\text { Clinical and/or } \\
\text { Radiologic DCI }\end{array}$ & $\begin{array}{l}\text { Patients } \\
\text { without } \\
\text { DCI (\%) }\end{array}$ & $P$ Value \\
\hline No. & 333 & $68(20)$ & $265(80)$ & - \\
\hline Female sex & $238(71)$ & $47(69)$ & $191(72)$ & .653 \\
\hline Age (yr) (mean) (SD) & 55.7 (11.9) & $56.0(12.7)$ & $55.7(11.7)$ & .828 \\
\hline Hypertension in medical history & $88(26)$ & $24(35)$ & $64(24)$ & .090 \\
\hline WFNS favorable on admission (I-III) & $163(49)$ & $34(50)$ & $129(49)$ & .892 \\
\hline Mean TBV (mL) (SD) ${ }^{\mathrm{a}}$ & $46.1(29.4)$ & $51.6(27.3)$ & $44.6(29.8)$ & .080 \\
\hline $\mathrm{IPH}$ & $102(31)$ & $16(24)$ & $86(32)$ & .185 \\
\hline $\mathrm{IVH}$ & $203(61)$ & $51(75)$ & $152(57)$ & .008 \\
\hline $\mathrm{IPH}$ and IVH & $63(19)$ & $12(18)$ & $51(19)$ & .863 \\
\hline Rebleed & $52(16)$ & $9(13)$ & $43(16)$ & .708 \\
\hline Anterior circulation & $245(74)$ & $50(74)$ & $195(74)$ & 1.000 \\
\hline Posterior circulation & $88(26)$ & $18(26)$ & $70(26)$ & \\
\hline Neurosurgical treatment ${ }^{\mathrm{b}}$ & $141(42)$ & $30(44)$ & $111(42)$ & .325 \\
\hline Endovascular treatment ${ }^{c}$ & $135(41)$ & $36(53)$ & $99(37)$ & \\
\hline Death within 3 days & $47(14)$ & $1(1)$ & 46 (17) & $<.001$ \\
\hline
\end{tabular}

${ }^{a}$ Automatically quantified total blood volume on noncontrast $\mathrm{CT}$.

${ }^{\mathrm{b}}$ Two patients treated with bypass surgery.

${ }^{c}$ One patient treated with stent, and 1 with parent vessel occlusion.

Table 2: Patient characteristics in groups with respect to DCI

\begin{tabular}{|c|c|c|c|c|}
\hline & $\begin{array}{l}\text { Patients with } \\
\text { Clinical and/or } \\
\text { Radiologic DCI }\end{array}$ & $\begin{array}{l}\text { Patients } \\
\text { with } \\
\text { Clinical } \\
\text { DCI (\%) }\end{array}$ & $\begin{array}{l}\text { Patients } \\
\text { with } \\
\text { Radiologic } \\
\text { DCl (\%) }\end{array}$ & $\begin{array}{l}\text { Patients with } \\
\text { Clinical and } \\
\text { Radiologic } \\
\text { DCI (\%) }\end{array}$ \\
\hline No. & $68(20)$ & $62(19)$ & $40(12)$ & $34(10)$ \\
\hline Female sex & $47(69)$ & $43(69)$ & $28(70)$ & 24 (71) \\
\hline Age (yr) (mean) (SD) & $56.0(12.7)$ & $55.4(12.9)$ & $57.9(12.4)$ & $57.1(12.8)$ \\
\hline Hypertension in medical history & $24(35)$ & $21(34)$ & $18(45)$ & $15(44)$ \\
\hline WFNS favorable on admission (I-III) & $34(50)$ & $30(48)$ & $18(45)$ & $14(41)$ \\
\hline Mean TBV $(\mathrm{mL})(\mathrm{SD})^{\mathrm{c}}$ & $51.6(27.3)$ & $52.1(27.4)$ & $53.2(28.1)$ & $54.3(28.3)$ \\
\hline $\mathrm{IPH}$ & $16(24)$ & $14(23)$ & $7(18)$ & $5(15)$ \\
\hline $\mathrm{IVH}$ & $51(75)$ & $47(76)$ & $31(78)$ & $27(79)$ \\
\hline IPH and IVH & $12(18)$ & $10(16)$ & $5(13)$ & $3(9)$ \\
\hline Rebleed & 9 (13) & $9(15)$ & $6(15)$ & $6(18)$ \\
\hline Anterior circulation & $50(74)$ & $45(73)$ & $28(70)$ & $23(68)$ \\
\hline Posterior circulation & $18(26)$ & $17(27)$ & $12(30)$ & $11(32)$ \\
\hline Neurosurgical treatment $^{d}$ & $30(44)$ & $28(45)$ & $19(48)$ & $17(50)$ \\
\hline Endovascular treatment ${ }^{\mathrm{e}}$ & $36(53)$ & $33(53)$ & $19(48)$ & $16(47)$ \\
\hline Death within 3 days & $1(1)$ & $1(2)$ & $1(3)$ & $1(3)$ \\
\hline
\end{tabular}

${ }^{a}$ Clinical $D C l$ with or without radiologic $D C l$.

${ }^{b}$ Radiologic DCl with or without clinical DCI.

${ }^{c}$ Automatically quantified total blood volume on noncontrast CT.

${ }^{\mathrm{d}}$ Two patients treated with bypass surgery.

e One patient treated with stent, and 1 with parent vessel occlusion.

ence of an intraparenchymal hematoma was negatively associated with DCI.

The association between TBV and DCI may appear small with an aOR of 1.02 per milliliter of blood. However, considering that the aOR is per milliliter of blood and that in our population, the mean TBV was $46.1 \mathrm{~mL}$ and the SD was almost $30 \mathrm{~mL}$, this effect is substantial: A difference of $1 \mathrm{SD}$ of TBV $(30 \mathrm{~mL})$ corresponds to an aOR of 1.81 .

In both analyses assessing the association between TBV and DCI, we found similar aORs for all 3 outcome variables (clinical DCI, radiologic DCI, and both). This finding could justify using 1 outcome variable in future studies. Clinical DCI would be the most appropriate to use because almost all patients with radiologic DCI have clinical DCI. Moreover, the importance of radiologic DCI in the absence of clinical DCI is questionable. CT or MR imaging might be performed for other reasons, showing areas of ischemia that are clinically unnoticed.

In the first publication on the relation between the amount and distribution of subarachnoid blood detected on NCCT and cerebral vasospasm (detected on angiography), it was concluded that blood localized in the subarachnoid space in sufficient amounts at specific sites is the only important etiologic factor in vasospasm. ${ }^{4}$ Because in our study we found an association of TBV, IPH, and IVH with clinical and radiologic DCI and not vasospasm, these studies are difficult to compare. One large difference is that in their study, not 1 patient with IVH developed clinical symptoms of DCI.

Only 1 more recent study investigated a semiautomatic blood quantification to assess the association between cisternal blood volume on NCCT and vasospasm after aSAH. ${ }^{16}$ In this study, a positive association was found. However, the method used was laborious because all blood was outlined manually. Moreover, no correction for potential confounders was performed.

Our study results are in line with results from other studies showing that patients without intraventricular blood and with a small amount of cisternal blood after aSAH are less likely to develop DCI, though the results are somewhat different because these studies used vasospasm as an end point instead of DCI. ${ }^{6,7}$ Nevertheless, these studies used the modified Fisher score instead of quantified blood volume to assess the amount of intracranial blood. ${ }^{4}$

The positive association between IVH and DCI is not yet understood. Blood can migrate toward the ventricles in 2 ways: first, straight from the aneurysm into the ventricles through a connecting hematoma or, second, by expansion of the subarachnoid blood toward one of the cisternal-ventricular foramina (Luschka, Magendie). The latter implies an initially higher volume of subarachnoid blood, with secondary ventricular redistribution. This might explain the association of IVH and DCI in this specific population. Additionally, patients who present with a nonaneurysmal IVH have a very low risk of developing DCI according to a study describing a series of patients with ruptured arteriovenous malformations, of whom 50 had an intraventricular component. Only 1 patient who also had an SAH component developed vasospasm, without signs of DCI. ${ }^{17}$ According to these and our results, it seems that the combination of IVH and aSAH is worse than IVH or aSAH alone.

When one tries to explain the negative association of the pres-

AJNR Am J Neuroradiol 37:1588-93 Sep 2016 www.ajnr.org 
Table 3: Associations between blood volume and blood location and DCI in the total group $(N=333)$

\begin{tabular}{lccc}
\hline \multicolumn{1}{c}{ Dependent Variable } & $\begin{array}{c}\text { Central } \\
\text { Determinant }\end{array}$ & OR (95\% CI) & aOR (95\% CI) \\
\hline Clinical DCI (with or without radiologic DCI) & $\mathrm{TBV}^{\mathrm{a}}$ & $1.01(1.0-1.02)^{\mathrm{b}}$ & $1.02(1.01-1.03)^{\mathrm{b}}$ \\
& $\mathrm{IPH}^{\mathrm{c}}$ & $0.61(0.32-1.16)$ & $0.47(0.24-0.95)$ \\
& $\mathrm{IVH}^{\mathrm{d}}$ & $2.31(1.23-4.33)$ & $2.66(1.37-5.17)$ \\
Radiologic DCI (with or without clinical DCI) & $\mathrm{TBV}^{\mathrm{e}}$ & $1.01(1.0-1.02)^{\mathrm{b}}$ & $1.02(1.01-1.03)^{\mathrm{b}}$ \\
Clinical and radiologic DCI & $\mathrm{TBV}^{\mathrm{f}}$ & $1.01(1.0-1.02)^{\mathrm{b}}$ & $1.02(1.01-1.03)^{\mathrm{b}}$ \\
\hline
\end{tabular}

${ }^{a}$ Confounders: age, WFNS, treatment, IPH, and IVH.

${ }^{\mathrm{b}}$ Per milliliter of blood.

${ }^{c}$ Confounder: treatment.

${ }^{\mathrm{d}}$ Confounders: WFNS and blood volume.

e Confounders: age, treatment, IPH, and IVH.

${ }^{f}$ Confounder: treatment, IPH, and IVH.

Table 4: Associations between blood volume and blood location and DCI in patients who survived 3 days or more $(n=286)$

\begin{tabular}{lccc}
\hline \multicolumn{1}{c}{ Dependent Variable } & $\begin{array}{c}\text { Central } \\
\text { Determinant }\end{array}$ & OR (95\% CI) & aOR (95\% CI) \\
\hline Clinical DCI (with or without radiologic DCI) & TBV $^{\mathrm{a}}$ & $1.01(1.00-1.03)^{\mathrm{b}}$ & $1.02(1.01-1.03)^{\mathrm{b}}$ \\
& $\mathrm{IPH}^{\mathrm{c}}$ & $0.65(0.33-1.25)$ & $0.43(0.21-0.89)$ \\
& $\mathrm{IVH}^{\mathrm{d}}$ & $2.41(1.27-4.57)$ & $2.03(1.05-3.92)$ \\
Radiologic DCI (with or without clinical DCI) & $\mathrm{TBV}^{\mathrm{a}}$ & $1.01(1.00-1.03)^{\mathrm{b}}$ & $1.02(1.01-1.03)^{\mathrm{b}}$ \\
Clinical and radiologic DCI & $\mathrm{TBV}^{\mathrm{a}}$ & $1.02(1.00-1.03)^{\mathrm{b}}$ & $1.02(1.01-1.03)^{\mathrm{b}}$ \\
\hline
\end{tabular}

${ }^{a}$ Confounders: IPH and IVH.

${ }^{\mathrm{b}}$ Per milliliter of blood.

${ }^{\mathrm{C}}$ Confounders: WFNS, blood volume, and IVH.

${ }^{d}$ Confounder: blood volume.

ence of an IPH with clinical DCI, it could very well be that in these patients, clinical DCI was less often detected because they already had a neurologic deficit due to the IPH. To our knowledge, there is no literature confirming this theory. Further studies are needed to confirm this and to determine the association between IVH and IPH and DCI. In such a study, blood volume values for each separate compartment can be determined and subsequently associated with the clinical course.

The strengths of our study are fast and objective estimation of the TBV by using a validated automatic quantification method, adjustment for confounders to make the estimation of the association more reliable, and performance of a sensitivity analysis to evaluate the robustness of the model. In addition, we used DCI as an outcome variable instead of vasospasm because DCI is a more clinically relevant outcome measure and because vasospasm and DCI can occur independently. ${ }^{2,12}$ In future studies, it might be possible to use the automated quantification method to study other subtypes of SAH (eg, due to intracranial dissection, AVM, benign perimesencephalic hemorrhage, and trauma).

Our study has some limitations. This was a retrospective study in which we could only study the data that were available in the clinical charts and on the available NCCTs. Because patients were followed up only during hospitalization, we may have potentially missed patients with DCI after discharge. In patients with clinical rebleeding within 24 hours after aSAH ictus, we might have underestimated the blood volume because this rebleeding was not CT-confirmed. Death during admission may be a competing risk for the development of DCI. Unfortunately, due to the composition of our data, we were unable to perform a reliable competing risk analysis.

Limitations of the automated quantification method are that imaging artifacts can make the results unreliable. Although we do not expect differences in the detection rate of TBV and aSAH between the anterior and posterior circulations, we have not yet investigated this possibility. Very small blood volumes with low density are not well-detected with automated detection. ${ }^{10}$ The volume assessments were performed on relatively thick sections of 5 $\mathrm{mm}$. Thinner sections could potentially increase accuracy. However, with thinner sections, the noise level increases as well, which may actually reduce the accuracy. The method was validated by using $5-\mathrm{mm}$ sections, and $5 \mathrm{~mm}$ is the standard section thickness used in our hospital and the referring centers. The volume of the IPH and IVH and the blood volume in separate territories could not be separately delineated.

\section{CONCLUSIONS}

We show that a higher TBV, measured with our automated quantification method, is significantly associated with DCI. The results of this study encourage the use of rater-independent quantification methods in future multicenter studies on DCI prevention and prediction.

Disclosures: Henk A. Marquering—RELATED: Grant: NutsOhra, ${ }^{*}$ Comments: The evaluation of the prognostic value of the quantitative hemorrhage volume detection is being sponsored by a grant from NutsOhra; UNRELATED: Grants/Grants Pending: NutsOhra.* Rene van den Berg-UNRELATED: Consultancy: DePuy Codman, Comments: coil development. Charles B. Majoie-UNRELATED: Grants/ Grants Pending: Dutch Heart Foundation,* Toegepast Wetenschappelijk Instituut voor Neuromodulatie Foundation*; Payment for Lectures (including service on Speakers Bureaus): Stryker. * Money paid to the institution.

\section{REFERENCES}

1. Budohoski KP, Guilfoyle M, Helmy A, et al. The pathophysiology and treatment of delayed cerebral ischaemia following subarachnoid haemorrhage. J Neurol Neurosurg Psychiatry 2014;85:1343-53 CrossRef Medline

2. Cossu G, Messerer M, Oddo M, et al. To look beyond vasospasm in aneurysmal subarachnoid haemorrhage. Biomed Res Int 2014;2014: 628597 CrossRef Medline

3. Macdonald RL. Delayed neurological deterioration after subarachnoid haemorrhage. Nat Rev Neurol 2014;10:44-58 CrossRef Medline

4. Fisher CM, Kistler JP, Davis JM. Relation of cerebral vasospasm to subarachnoid hemorrhage visualized by computerized tomographic scanning. Neurosurgery 1980;6:1-9 CrossRef Medline

5. Frontera JA, Claassen J, Schmidt JM, et al. Prediction of symptomatic vasospasm after subarachnoid hemorrhage: the modified Fisher scale. Neurosurgery 2006;59:21-27, discussion 21-27 Medline

6. Claassen J, Bernardini GL, Kreiter K, et al. Effect of cisternal and ventricular blood on risk of delayed cerebral ischemia after subarachnoid hemorrhage: the Fisher scale revisited. Stroke 2001;32: 2012-20 CrossRef Medline

7. de Rooij NK, Greving JP, Rinkel GJ, et al. Early prediction of delayed cerebral ischemia after subarachnoid hemorrhage: development and validation of a practical risk chart. Stroke 2013;44:1288-94 CrossRef Medline 
8. de Rooij NK, Rinkel GJ, Dankbaar JW, et al. Delayed cerebral ischemia after subarachnoid hemorrhage: a systematic review of clinical, laboratory, and radiological predictors. Stroke 2013;44:43-54 CrossRef Medline

9. Crobeddu E, Mittal MK, Dupont S, et al. Predicting the lack of development of delayed cerebral ischemia after aneurysmal subarachnoid hemorrhage. Stroke 2012;43:697-701 CrossRef Medline

10. Boers AM, Zijlstra IA, Gathier CS, et al. Automatic quantification of subarachnoid hemorrhage on noncontrast CT. AJNR Am J Neuroradiol 2014;35:2279-86 CrossRef Medline

11. Report of World Federation of Neurological Surgeons Committee on a Universal Subarachnoid Hemorrhage Grading Scale. J Neurosurg 1988;68:985-86 Medline

12. Vergouwen MD. Vasospasm versus delayed cerebral ischemia as an outcome event in clinical trials and observational studies. Neurocrit Care 2011;15:308-11 CrossRef Medline
13. Yushkevich PA, Piven J, Hazlett HC, et al. User-guided 3D active contour segmentation of anatomical structures: significantly improved efficiency and reliability. Neuroimage 2006;31:1116-28 CrossRef Medline

14. van der Zande JJ, Hendrikse J, Rinkel GJ. CT angiography for differentiation between intracerebral and intra-Sylvian hematoma in patients with ruptured middle cerebral artery aneurysms. AJNR Am J Neuroradiol 2011;32:271-75 CrossRef Medline

15. Twisk JW. Inleiding in de toegepaste biostatistiek. Vol 3. Maarssen: Elsevier Gezondheidszorg; 2014:242

16. Friedman JA, Goerss SJ, Meyer FB, et al. Volumetric quantification of Fisher grade 3 aneurysmal subarachnoid hemorrhage: a novel method to predict symptomatic vasospasm on admission computerized tomography scans. J Neurosurg 2002;97:401-07 CrossRef Medline

17. Gross BA, Du R. Vasospasm after arteriovenous malformation rupture. World Neurosurg 2012;78:300-05 CrossRef Medline 